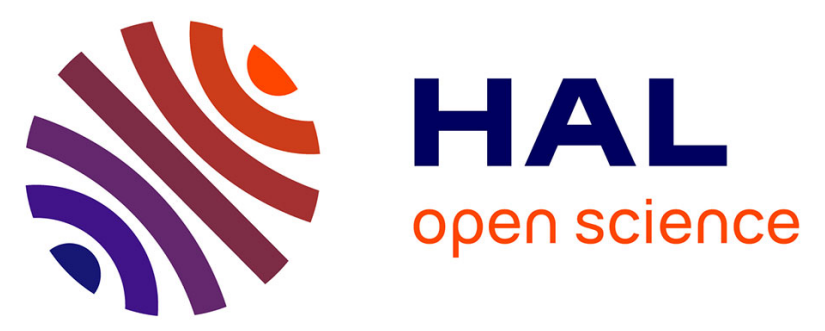

\title{
Limitations and performances of robust control over WSN: UFAD control in intelligent buildings
}

Emmanuel Witrant, Piergiuseppe Di Marco, Pangun Park, Corentin Briat

\section{To cite this version:}

Emmanuel Witrant, Piergiuseppe Di Marco, Pangun Park, Corentin Briat. Limitations and performances of robust control over WSN: UFAD control in intelligent buildings. IMA Journal of Mathematical Control and Information, 2010, 27 (4), pp.527-543. 10.1093/imamci/dnq017 . hal-00562164

\section{HAL Id: hal-00562164 \\ https://hal.science/hal-00562164}

Submitted on 2 Feb 2011

HAL is a multi-disciplinary open access archive for the deposit and dissemination of scientific research documents, whether they are published or not. The documents may come from teaching and research institutions in France or abroad, or from public or private research centers.
L'archive ouverte pluridisciplinaire HAL, est destinée au dépôt et à la diffusion de documents scientifiques de niveau recherche, publiés ou non, émanant des établissements d'enseignement et de recherche français ou étrangers, des laboratoires publics ou privés. 


\title{
Limitations and Performances of Robust Control over WSN: UFAD Control in Intelligent Buildings
}

\author{
EMMANUEL WITRANT \\ Grenoble Image Parole Signal Automatique (GIPSA-lab), \\ University Joseph Fourier, FR-38402 Saint Martin d'Hères, France \\ Corresponding author: emmanuel.witrant@gipsa-lab.inpg.fr \\ PIERGIUSEPPE DI MARCO, PANGUN PARK \\ ACCESS Linnaeus Center, Department of Electrical Engineering, \\ KTH - Royal Institute of Technology, SE-100 44 Stockholm, Sweden \\ pidm,pgpark@ee.kth.se \\ $A N D$ \\ CORENTIN BRIAT \\ ACCESS Linnaeus Center, Department of Mathematics, \\ KTH - Royal Institute of Technology, SE-100 44 Stockholm, Sweden \\ corentin@briat.info
}

\begin{abstract}
The aim of this paper is to propose a model-based feedback control strategy for indoor temperature regulation in buildings equipped with underfloor air distribution (UFAD). Supposing distributed sensing and actuation capabilities, a OD model of the ventilation process is derived, based on the thermodynamics properties of the flow. A state-space description of the process is then inferred, including discrete events and nonlinear components. The use of a wireless sensor network (WSN) and the resulting communication constraints with the IEEE 802.15.4 standard are discussed. Both synchronous and asynchronous transmissions are considered. Based on the linear part of the model, different $H_{\infty}$ robust multiple-input multiple-output (MIMO) controllers are designed, first with a standard mixed-sensitivity approach and then by taking into account the network-induced delay explicitly. The impact of the communication constraints and the relative performances of the controllers are discussed based on simulation results.
\end{abstract}

Keywords: Energy applications; Hybrid modeling; Network-controlled systems; Robust control.

\section{Introduction}

Intelligent buildings ventilation control is a challenging automation problem with objectives that rise several research problems of immediate actuality, such as the wireless automation and the control of complex interconnected subsystems. The system considered is composed of ventilated rooms, fans, plenums and a wireless network. The complexity arises from the different physical properties - and associated dynamics - of the subsystems. In a broader picture, all these engineering problems imply to deal with fluid models and the connection of different subsystems. Global control strategies are of prime importance to deal with such problems.

Recent results have illustrated the interest for under floor air distribution (UFAD) solutions in comparison with traditional ceiling-based ventilation, as mentioned by the Bauman and Daly (2003). An UFAD indoor climate regulation process is set with the injection of a fresh airflow from the floor and an exhaust located at the ceiling level, as depicted in Figure 1(a). Note that we consider the specific case 


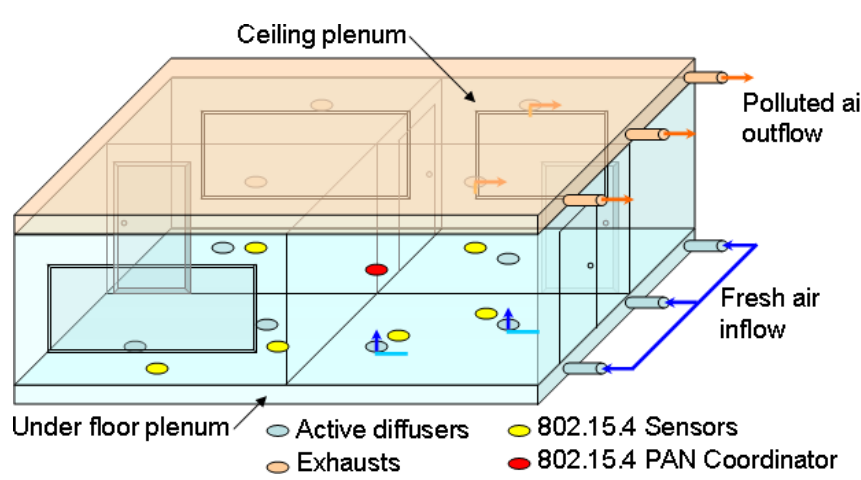

(a) Overview with automation devices

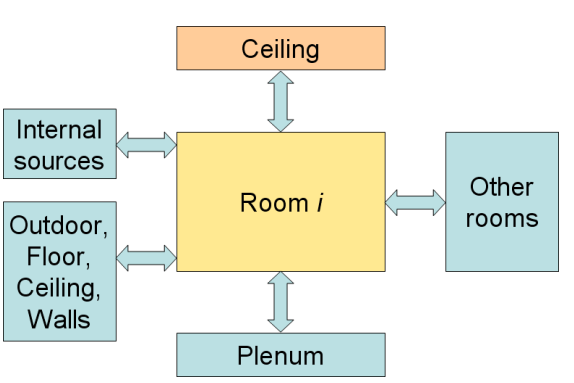

(b) Bloc-diagram model

FIG. 1. Under floor air distribution

where a common plenum is used at both the underfloor and ceiling levels. It has been established that well-designed UFAD systems can reduce life-cycle building costs, improve thermal comfort, ventilation efficiency and indoor air quality, conserve energy, and reduce floor-to-floor height. Feedback regulation, as considered in this paper, is a key element for an optimized system operation and it can be achieved thanks to actuated diffusers and distributed measurements provided by a wireless sensor network (WSN) deployed in the ventilated area.

Global regulation strategies are particularly difficult to establish for such plants, due to the system complexity and the real time constraints. In order to set a model-based control approach, we first investigate the thermodynamics properties of the ventilation process with a control volume approach. We will show that it allows for a reduced-order, easily reconfigurable system description but implies nonlinearities and discrete events (doors, internal power sources, etc.). Such events are handled specifically with an Markovian approach and the resulting system is described as a hybrid state-space model. The distributed sensing capabilities associated with the WSN are considered by proposing a specific network architecture and highlighting the related communication constraints (bandwidth limitation and time delays).

The purpose of this paper is to describe the main dynamics associated with UFAD feedback regulation and illustrate the use of the resulting model in a simple control strategy, including the communication constraints induced by the WSN. In this sense, it is not aimed here to design a controller which will vary according to the stochastic processes (i.e. a multi-mode controller) but actually to ensure that the control strategy will be robust enough to cope with variations and disturbances due to Markovian processes, time-delays and nonlinearities. The $H_{\infty}$ control approach has then be selected due to its intrinsic robustness property, a systematic design procedure, and its efficiency in a wide range of applications, in particular with time-delays as illustrated in Sename and Fattouh (2007). Other types of controller can be synthesized based on bounded-real lemmas for linear time-invariant (LTI) systems without delays (Skelton et al., 1997) and for systems with time-varying sampling period. Indeed, since the delay induced by the network has a sawtooth shape, then such a problem can be attacked with methods described in (Fridman et al., 2004; Naghshtabrizi et al., 2008; Seuret, 2009). Our aim is to investigate the capability of such linear control approaches to cope with the stochastic and nonlinear behavior of the system. A mixed sensitivity $H_{\infty}$ synthesis is then compared with two approaches that explicitly take into account the communication constraints (expressed as time-delays).

The paper is organized as follows. First, the UFAD process is described as an interconnected model in Section 2. This model is used in Section 3 to obtain a hybrid state-space description, including the 
Markovian processes. Section 4 details the deployment of the WSN and the impact of the IEEE 802.15.4 standard. The design of a $H_{\infty}$ controller based on a mixed-sensitivity approach is described in Section 5. The synthesis of controllers thanks to bounded-real lemmas and the explicit consideration of the delays are detailed in Section 6.

\section{UFAD energy-consistent model}

In order to provide a model that is suited for real-time feedback control, we consider a 0-D model based on the mass and energy conservation in each room. Each room is interconnected with the other building elements, as depicted in Figure 1(b). The interconnections are fully defined, for the choice of any couple of components, by the mass flow rate and heat transport. A generic and flexible model of an UFAD system is thus obtained thanks to an appropriate classification of the mass and heat sources, combined with the continuity equation and the first and second laws of thermodynamics in each room. In comparison with more thorough models such as EnergyPlus ${ }^{\mathrm{TM}}$ (US department of energy, 2008), our approach is focused on the flexibility of its application and on the key dynamics for control purposes.

\subsection{Continuity and conservation of energy}

The room dynamics is set using the fundamental laws of thermodynamics, described in classical textbooks such as Sonntag et al. (1998). The internal state of room $i$, described by its density and temperature $<\rho_{i}, T_{i}>$, is determined based on the following hypotheses:

H1. the flow is incompressible: $\rho_{i}=\rho_{\text {air }}$;

H2. the control volume $(\mathrm{CV})$ remains constant relative to the coordinate frame;

H3. the state of mass within the $\mathrm{CV}$ is uniform at any time;

H4. the state of the mass crossing the $\mathrm{CV}$ is constant with time (the mass flow rates may vary);

H5. the kinetic and potential energy of the gas within the room are neglected.

The first assumption is straightforward considering the low speed of the airflow within the rooms. (H2)(H4) are classical for uniform-state, uniform-flow processes, i.e. when there is no change in the state of mass (we consider only gas in our case). (H5) is associated with the fact that the gas moves slowly in the room and that the mass of the gas in the $\mathrm{CV}$ is not important enough to generate significant potential energy. The CV considered is strictly limited to the inside room volume: it does not include the underfloor and ceiling plenums.

The continuity equation, along with incompressibility $(H 1)$, implies that the mass conservation writes as $\sum \dot{m}_{i n_{i}}=\sum \dot{m}_{\text {out }}$, where $\dot{m}_{\text {in }}$ and $\dot{m}_{\text {out }}$ are the input and output mass flow rates, respectively. The first law of thermodynamics, applied with $(H 2)-(H 4)$ and without mechanical work, gives the energy exchange in the room $\mathrm{CV}$ as:

$$
\frac{d E_{i}}{d t}=\dot{Q}_{i}+\sum \dot{m}_{\text {in }} h_{\text {tot }, \text { in }}-\sum \dot{m}_{\text {out }} h_{\text {tot }, \text { out }} \text { i }
$$

where $E_{i}$ is the room energy, $\dot{Q}_{i}$ the heat exchange and $h_{\text {tot }}$ the total enthalpy, approximated as $h_{t o t}=C_{p} T$ with $C_{p}$ the constant pressure specific heat. Considering (H5), the room energy is the internal energy (constant volume) and $E_{i}=\rho_{a i r} V_{i} C_{v} T_{i}$, where $V_{i}$ is the room volume and $C_{v}$ is the constant volume specific heat. For air at $25^{\circ} \mathrm{C}$ and $1 \mathrm{~atm}, C_{v}=717 \mathrm{~J} / \mathrm{kg} . \mathrm{K}, C_{p}=1004 \mathrm{~J} / \mathrm{kg} . \mathrm{K}$ and $\rho_{\text {air }}=1.169 \mathrm{~kg} / \mathrm{m}^{3}$.

The heat exchange $Q_{i}$ can be decomposed, depending on the nature of the heat transfers, as: 


\begin{tabular}{|ll|}
\hline Component & Associated energy \\
\hline Inside walls $i w$ & $-k_{i w} A_{i w}\left(T_{i}-T_{j}\right) / \Delta x_{i w}$ \\
Outside walls ow & $-\left(k_{\text {ow }} \frac{A_{\text {ow }}}{\Delta x_{o w}}+k_{\text {glass }} \frac{A_{\text {glass }}}{\Delta x_{\text {glass }}}\right)\left(T_{i}-T_{\text {out }}\right)$ \\
Plenum $p l$ & $C_{p} \dot{m}_{p l} T_{p l}$ \\
Floor & $-k_{p l} A_{p l}\left(T_{i}-T_{p l}\right) / \Delta x_{p l}$ \\
Ceiling $c$ & $-C_{p} \dot{m}_{c} T_{i}$ \\
People $b$ & $\varepsilon \sigma A_{b}\left(T_{b}^{4}-T_{i}^{4}\right)$ \\
Inside sources & $\dot{Q}_{\text {sources }}$ \\
Doors $d$ & $C_{p} \rho A_{d} \sqrt{2 R\left(T_{j}-T_{i}\right)} T_{j}$, if $T_{j}>T_{i}$ \\
& $C_{p} \rho A_{d} \sqrt{2 R\left(T_{i}-T_{j}\right)} T_{i}$, if $T_{i}>T_{j}$ \\
\hline
\end{tabular}

Table 1. Energy sources in room $i$.

- conduction (Fourier's law): $\dot{Q}_{\text {cond }}=k A \Delta T / \Delta x$, where $k[W / m . K]$ is the conductivity $(\approx 10$ for glass, 0.1 for insulation materials) and $A$ the surface area where exchanges occur;

- convection (Newton's law): $\dot{Q}_{\text {conv }}=A h \Delta T$, where $h\left[W / m^{2}\right]$ is the heat transfer coefficient (typically within the range 5-25 for natural convection and 25-250 for forced convection, sometimes expressed in $\left.\left[W / m^{2} \cdot K\right]\right)$;

- radiation (electromagnetic waves): $\dot{Q}_{\text {rad }}=\varepsilon \sigma A T_{s}^{4}$, where $\varepsilon$ is the emissivity ( 0.92 for nonmetalic surfaces), $\sigma=5.67 \times 10^{-8} \mathrm{Wm}^{-2} \mathrm{~K}^{-4}$ is the Stephan-Boltzmann constant and $T_{s}$ is the surface temperature.

Under the previous hypotheses, the mass flow rate $\dot{m}$ going from a high temperature volume $T_{h}$ to a low temperature volume $T_{l}$ through a section $A$ is obtained by combining Bernoulli's and the ideal gas equations as:

$$
\dot{m}=\rho A \sqrt{2 R\left(T_{h}-T_{l}\right)}
$$

where $R=C_{p}-C_{v}$.

\subsection{Room dynamics}

Based on the previous description, we obtain the room temperature dynamics:

$$
\frac{d T_{i}}{d t}=\frac{1}{\rho_{\text {air }} V_{i} C_{v}}\left[\dot{Q}_{\text {conv }}+\dot{Q}_{\text {cond }}+\dot{Q}_{\text {rad }}+\dot{Q}_{\text {sources }}+C_{p} \sum \dot{m}_{\text {in }_{i}} T_{\text {in }_{i}}-C_{p} \sum \dot{m}_{\text {out }} T_{i}\right]
$$

where we introduced the additional source $\dot{Q}_{\text {sources }}$ to model the internal heat sources (computers, printers, etc.) and considered the outflow temperature as the room temperature (which is a direct consequence of the 0-D approximation). A simplified classification of the heat sources for room $i$ is proposed in Table 1 , where $T_{j}$ indicates the temperature in an adjacent room, $A_{x}$ the exchange surface areas and $\Delta x_{x}$ the thicknesses. Note that the last three components correspond to discrete events while the previous ones have continuous variations. This description is easily refined by introducing additional terms (walls radiation, windows airflow, etc.), depending on the desired level of model accuracy.

The ceiling, plenum and doors mass flow rates are constrained by the conservation of mass (continuity) with (setting $\dot{m}>0$ when the flow is entering the room):

$$
\dot{m}_{c_{i}}+\sum_{l=1 \ldots N_{p l}} \dot{m}_{p l_{i}, l}+\sum_{l=1 \ldots N_{d}} \dot{m}_{d_{i j}, l}=0
$$


where $N_{p l}$ is the number of diffusers in the room and $N_{d}$ denotes the number of doors. The doors mass flow rate can be computed thanks to Bernouilli equation (2.1) as $\dot{m}_{d}=\operatorname{sign}\left(T_{j}-T_{i}\right) \rho A_{d} \sqrt{2 R\left|T_{j}-T_{i}\right|}$, where the sign function is introduced to indicate the flow direction. The temperature regulation is achieved by controlling the mass flow rate from the plenum $\dot{m}_{p l_{i}, l}(t)$, considering a given underfloor temperature $T_{p l}(t)$ (regulated globally for the whole building). We will suppose in the following that there is only one diffuser per room $\left(N_{p l}=1\right)$ and that the WSN provides the temperature measurements for the feedback law.

Finer models, including the height-dependency of the temperature variations, can be derived using the stratified flow theory (Morton, 1959; Yih, 1969) or buoyancy driven flow dynamics (Gladstone and Woods, 2001). The WSN measurements can also be set to determine the temperatures distribution shape, along the lines suggested in Witrant et al. (2010a). The use of a coarse global model based on the proposed 0-D approach is motivated by the fact that the WSN directly provides the necessary measurements for feedback control. The aim of the model is then to give the proper directions of the regulated states according to the actuation and disturbances.

\section{Hybrid state-space model}

\subsection{Continuous dynamics}

The continuous dynamics of the model is set by the walls, ceiling and plenum. According to the physical laws described in Section 2.2 and supposing that all the doors are closed and that there is no power source within the room, the temperature dynamics for room $i$ is given as:

$$
\begin{aligned}
\frac{d T_{i}}{d t}= & \frac{1}{\rho_{\text {air }} V_{i} C_{v}}\left[\dot{Q}_{\text {conv }}+\dot{Q}_{c o n d}+\dot{Q}_{\text {rad }}+C_{p} \dot{m}_{p l_{i}}\left(T_{p l}-T_{i}\right)\right] \doteq F_{c i}(t) \\
= & \frac{1}{\rho_{\text {air }} V_{i} C_{v}}\left[-\sum_{l=1 \ldots N_{i w}} \alpha_{i w_{l}}\left(T_{i}-T_{l}\right)-\sum_{l=1 \ldots N_{o w}}\left(\alpha_{o w_{l}}+\alpha_{\text {glass }_{l}}\right)\left(T_{i}-T_{\text {out }}\right)+C_{p} \dot{m}_{p l_{i}} T_{p l}\right. \\
& \left.-\alpha_{p l_{i}}\left(T_{i}-T_{p l}\right)-C_{p} \dot{m}_{c} T_{i}\right] \\
= & \frac{1}{\rho_{a i r} V_{i} C_{v}}\left[-\left(\Xi_{i}+C_{p} \dot{m}_{p l_{i}}\right) T_{i}+\sum_{l=1 \ldots N_{i w}} \alpha_{i w_{l}} T_{l}+\sum_{l=1 \ldots N_{o w}}\left(\alpha_{o w_{l}}+\alpha_{\text {glass }_{l}}\right) T_{\text {out }}\right. \\
& \left.+\left(C_{p} \dot{m}_{p l_{i}}+\alpha_{p l_{i}}\right) T_{p l}\right]
\end{aligned}
$$

where $\Xi_{i} \doteq \sum_{l=1 \ldots N_{i w}} \alpha_{i w_{l}}+\sum_{l=1 \ldots N_{o w}}\left(\alpha_{o w_{l}}+\alpha_{\text {glass }}\right)+\alpha_{p l_{i}}, \alpha_{x}=k_{x} A_{x} / \Delta x_{x}$ for component $x, N_{i w}$ is the number of connected inside walls, $N_{o w}$ is the number of outside walls, and $F_{c i}(t)$ denotes the continuous part of the dynamics for room $i$.

Defining the state as the vector containing the rooms temperatures $x=\left[\begin{array}{lll}T_{1} & T_{2} \ldots T_{n}\end{array}\right]^{T}$, the controlled input as $u \doteq\left[\begin{array}{llll}\dot{m}_{p l_{1}} & \dot{m}_{p l_{2}} \ldots \dot{m}_{p l_{n}}\end{array}\right]^{T}$ and the exogenous input as $w \doteq\left[\begin{array}{ll}T_{p l} & T_{\text {out }}\end{array}\right]^{T}$, where $n$ is the number of rooms, the system dynamics writes as:

$$
\frac{d x}{d t}=\left(A_{1}+A_{2}(u)\right) x+B_{u} u+B_{w} w \doteq f_{c}(x, u, w)
$$

where the state matrices $A_{1,2}$ and the input matrices $B_{u, w}$ are computed according to (3.1). The function $f_{c}$ is introduced to denote the continuous part of the model in a compact form. Note that this model is fully determined by the building architecture and constant physical variables. 


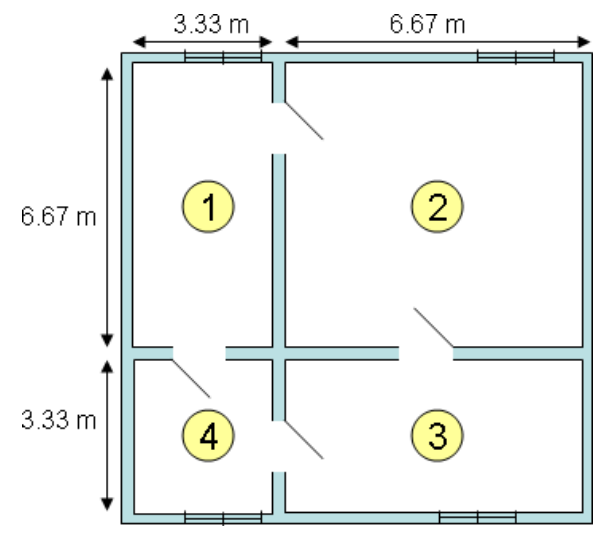

FIG. 2. Flat architecture for the test case

EXAMPLE 3.1 We consider the 4-rooms flat architecture presented in Figure 2: each room has a different size, two doors (two neighboring rooms) and some windows. The plenum temperature $T_{p l}$ is given, the state is $x=\left[T_{1} \ldots T_{4}\right]^{T}$, the controlled input is $u=\left[\dot{m}_{p l_{1}} \ldots \dot{m}_{p l_{4}}\right]^{T}$ and the exogenous input is $w=\left[T_{p l} T_{\text {out }}\right]^{T}$. The state and inputs matrices are obtained as:

$$
\begin{aligned}
& A_{1}=\left[\begin{array}{cccc}
a_{11}^{1} & a_{12}^{1} & 0 & a_{14}^{1} \\
a_{21}^{1} & a_{22}^{1} & a_{23}^{1} & 0 \\
0 & a_{32}^{1} & a_{33}^{1} & a_{34}^{1} \\
a_{41}^{1} & 0 & a_{43}^{1} & a_{44}^{1}
\end{array}\right], \quad B_{w}=\left[\begin{array}{cc}
b_{1}^{w 1} & b_{1}^{w 2} \\
b_{2}^{w 1} & b_{2}^{w 2} \\
b_{3}^{w 1} & b_{3}^{w 2} \\
b_{4}^{w 1} & b_{4}^{w 2}
\end{array}\right], \\
& A_{2}(u)=\operatorname{diag}\left(a_{i}^{2}\right) \cdot \operatorname{diag}\left(u_{i}\right), \quad B_{u}=-\operatorname{diag}\left(a_{i}^{2}\right) \cdot T_{p l} \text {, }
\end{aligned}
$$

with:

$$
\begin{gathered}
a_{i i}^{1}=-\frac{\Xi_{i}}{\rho_{a i r} V_{i} C_{v}}, \quad a_{i j}^{1}=\frac{\alpha_{i w, i j}}{\rho_{a i r} V_{i} C_{v}} \quad i \neq j, \quad a_{i}^{2}=-\frac{C_{p}}{\rho_{a i r} V_{i} C_{v}} \\
b_{i}^{w 1}=\frac{\alpha_{p l_{i}}}{\rho_{\text {air }} V_{i} C_{v}}, \quad b_{i}^{w 2}=\frac{1}{\rho_{a i r} V_{i} C_{v}} \sum_{l=1 \ldots N_{\text {ow }}}\left(\alpha_{o w_{l}}+\alpha_{\text {glass }_{l}}\right) .
\end{gathered}
$$

\subsection{Discrete events}

The discrete events are induced by the power sources and the doors. Concerning the doors influence, the proposed energy-based model implies that for a given room $i$ and adjacent room $j$ (supposing that there is no return from the upper plenum):

- if $T_{i}>T_{j}$ then the flow going out of room $i$ equals the inflow $\dot{m}_{p l_{i}}$ and the fact that it is leaving through the ceiling or the open door does not change the energy balance (the energy loss due to outflows being included in the dynamics with the assumption that the outgoing mass flow rate is equal to the incoming mass flow rate);

- if $T_{i}<T_{j}$ then an extra term $C_{p} \dot{m}_{d}\left(T_{j}-T_{i}\right)$ has to be introduced, with $\dot{m}_{d}=\rho A_{d} \sqrt{2 R\left(T_{j}-T_{i}\right)}$. 


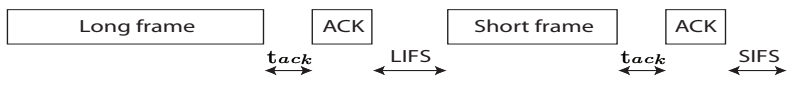

(a) Acknowledged transmission

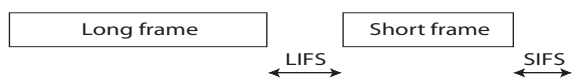

(b) Unacknowledged transmission

FIG. 3. IFS data transmission mechanism with and without acknowledgement.

The room temperature is then obtained as (supposing that only one door is open in room $i$ at a given time, to simplify the notations):

$$
\frac{d T_{i}}{d t}=F_{c i}(t)+\frac{1}{\rho_{a i r} V_{i} C_{v}} \times \begin{cases}\sum_{k=0}^{N_{q}} \delta_{q, i k} \dot{Q}_{s, i k}, & \text { if } T_{i}>T_{j} \\ \sum_{k=0}^{N_{q}} \delta_{q, i k} \dot{Q}_{s, i k}+\delta_{d, i j} C_{p} \sqrt{2 R}\left(T_{j}-T_{i}\right)^{3 / 2}, & \text { if } T_{i}<T_{j}\end{cases}
$$

where $N_{q}$ is the number of power sources, and $\delta_{q, i k}$ and $\delta_{d, i j}$ are introduced to denote the on/off operation of power sources and doors opening in room $i$. The discrete transitions are set by Markovian independent processes (e.g. finite birth and death) constrained by the maximum and minimum periods during which a given event can occur. Further details are provided in Witrant et al. (2009).

EXAMPLE 3.2 For the 4-rooms example described previously, the temperature increase associated to the door opening event $\delta_{d, i j}$ is handled as:

$$
\text { If } T_{i}<T_{j} \text { then } \dot{T}_{i}=\dot{T}_{i}+C_{p} \sqrt{2 R}\left(T_{j}-T_{i}\right)^{3 / 2} \text {, else } \dot{T}_{j}=\dot{T}_{j}+C_{p} \sqrt{2 R}\left(T_{i}-T_{j}\right)^{3 / 2} \text {. }
$$

enclosed in a for loop to handle the 4 doors.

\section{Wireless sensor network}

In this section, we present an overview of IEEE 802.15.4 standard (IEEE, 2006) and the system scenario with simulation results, which are used in the next Sections to estimate the efficiency of the proposed control setup.

\subsection{Overview of IEEE 802.15.4}

The IEEE 802.15.4 standard has received considerable attention as a low data rate and low power protocol for WSN applications in industry, control, home automation, health care, and smart grids (IEEE, 2006; Willig et al., 2005). This standard specifies medium access control (MAC) and physical (PHY) layers. The carrier sense multiple access with collision avoidance (CSMA/CA) is used along with a binary exponential backoff (BEB) scheme to reduce collisions due to simultaneous node transmissions. The standard defines two channel access modalities: a beacon-enabled modality, which uses slotted CSMA/CA and exponential backoff, and a simpler unslotted CSMA/CA without beacons. In the following, we focus on the slotted modality.

Consider a node trying to transmit a packet. In slotted IEEE 802.15.4 CSMA/CA, first the MAC sub-layer initializes four variables, i.e., the number of backoffs $(\mathrm{NB}=0)$, contention window $(\mathrm{CW}=2)$, backoff exponent $(\mathrm{BE}=$ macMinBE $)$ and retransmission times $(\mathrm{RT}=0)$. Then the MAC sub-layer delays 
for a random number of complete backoff periods in the range $\left[0,2^{\mathrm{BE}}-1\right]$ units. After backoff, the node performs the first clear channel assessment (CCA). If two consecutive CCAs are idle, then the node begins the packet transmission. If either of the CCA fails due to busy channel, the MAC sublayer will increase the value of both NB and BE by one up to a maximum value macMaxCSMABackoffs and mac$M a x B E$, respectively. Hence, the value of NB and BE depend on the number of CCA failures of a packet. Once the BE reaches macMaxBE, it remains at the value of macMaxBE until it is reset. If NB exceeds macMaxCSMABackoffs, then the packet is discarded due to the channel access failure. Otherwise the CSMA/CA algorithm generates a random number of complete backoff periods and repeat the process. Here, the variable macMaxCSMABackoffs represents the maximum number of times the CSMA/CA algorithm is required to backoff. If channel access is successful, the node starts transmitting packets and waits for acknowledgement (ACK). The reception of the corresponding ACK is interpreted as successful packet transmission. If the node fails to receive ACK due to collision or ACK timeout, the variable RT is increased by one up to macMaxFrameRetries. If RT is less than macMaxFrameRetries, the MAC sublayer initializes two variables $\mathrm{CW}=0, \mathrm{BE}=$ macMinBE and follows the CSMA/CA mechanism to reaccess the channel. Otherwise the packet is discarded due to the retry limit. Note that the default MAC parameters are macMinBE=3, macMaxBE=5, macMaxCSMABackoffs=4 and macMaxFrameRetries=3.

To account for the data processing time required at the MAC sublayer, two successive frames transmitted from a device are separated by at least an Inter-Frame Spacing (IFS) period; if the first transmission requires an acknowledgment, the separation between the ACK frame and the second transmission is at least an IFS period. Figure 3 illustrates the IFS period of data frame with and without ACK. Note that the waiting time to receive ACK is in the range aTurnaroundTime (12 symbols) to aTurnaroundTime + aUnitBackoffPeriod $(12+20$ symbols). The IFS period depends on the length of the transmitted data frames. See IEEE (2006) for further details.

In conclusion, the standard gives a time-varying packet delay due to the random access scheme. It is possible to look at the source of packet delay for different frequency domains (Witrant et al., 2010b):

- high frequency delays, due to the packet transmission time;

- middle frequency delays, which depend on the random backoff time (macMinBE, macMaxBE) and the number of busy channel (macMaxCSMABackoffs);

- low frequency delays, related to the number of retransmission due to packet collision (macMaxFrameRetries).

\subsection{Analytical model of IEEE 802.15.4}

In Park et al. (2009), a generalized analysis of the IEEE 802.15.4 MAC protocol in terms of packet reception rate (reliability), delay and energy consumption is presented. The IEEE 802.15.4 exponential backoff process is modeled through a Markov chain taking into account retry limits, acknowledgements, and unsaturated traffic. Evaluating these performance indicators asks in general for heavy computations. As such, these expressions may not be directly applied to optimize the IEEE 802.15.4 MAC parameters by an in-network processing of the nodes (Giridhar and Kumar, 2006) since complex computations are out of reach for today's sensing devices (Moteiv, 2006). To overcome this problem, simple and effective approximations of the reliability, delay and energy consumption under low traffic regime are proposed with a satisfactory accuracy. Monte Carlo simulations validates the proposed analytical model. Hence, from a communication network perspective, we are able to predict accurately the reliability, packet delay, and energy consumption. 


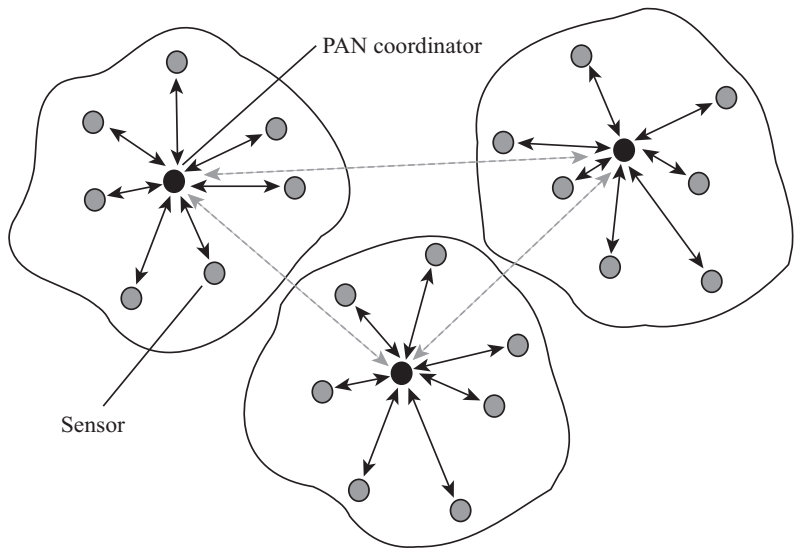

FIG. 4. Typical star network topology of IEEE 802.15.4. The packets generated by the sensor nodes (grey circle) are transmitted toward the PAN coordinator (black circle) depicted in the middle of each cluster.

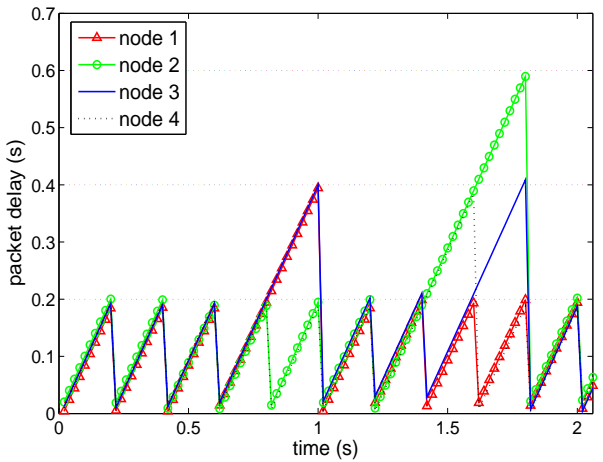

(a) Saw-tooth packet delay of Sync-mode

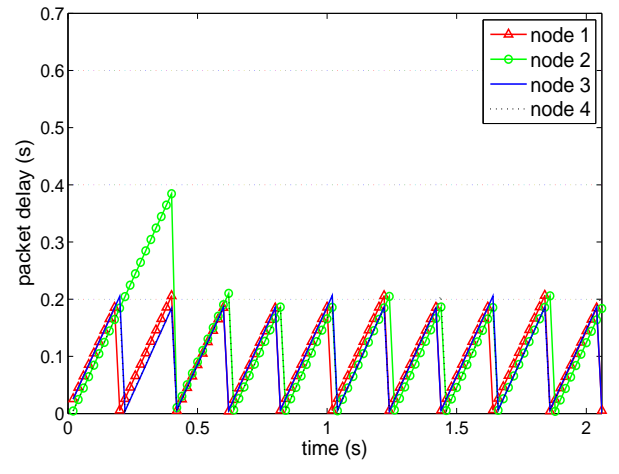

(b) Saw-tooth packet delay of Async-mode

FIG. 5. Saw-tooth packet delay with 70 bytes packets and four nodes for two traffic generation models.

\subsection{System scenario}

We consider the star network presented in Figure 4, where all $N$ nodes contend to send data to the personal area network (PAN) coordinator, which is the data sink attached to a controller. Each node uses beacon-enabled slotted CSMA/CA and ACK mechanism. Assume that the network generates unsaturated traffic, which is a natural scenario for many WSN applications.

Throughout this paper we refer to two models for traffic generation. Let us call by Sync-mode and Async-mode the situation when each node synchronously or asynchronously generate packets with the sampling interval $T_{s}(t)$ respectively. In Sync-mode, nodes of the network are synchronized and generate the data packets at the same time with a fixed sampling interval $T_{s}$. In Async-mode, each node asynchronously generates packets. When a node sends a packet successfully, discards a packet or when the sampling interval is expired, it stays for $T_{s} \mathrm{~s}$ without generating packet. Figure 5 shows the sawtooth packet delay of each node for two traffic generation models. The saw-tooth packet delay increases 
with the unit time $T_{u}$ until the PAN coordinator receives a data packet. If a data packet is received, the saw-tooth packet delay is reset to received packet delay. Therefore, the saw-tooth packet delay is able to represent the packet loss i.e., packet loss will increase the saw-tooth packet delay greater at a higher level than $T_{s}$. Figures 5(a) and 5(b) show the saw-tooth packet delay of Sync-mode and Asyncmode, respectively. We remark here that although both Sync-mode and Async-mode have almost the same time interval $T_{s}$, the reliability of Async-mode is higher than the one of Sync-mode. The reason is that Async-mode effectively distributes the sensing time of each sensor node based on the history of contention. It can be observed that the sampling time instant of Async-mode is spread for the different nodes of the network. The data packet transmission is successful if an ACK packet is received. The command on control action is piggybacked on ACK packet and does not require any additional message from the PAN coordinator to sensor nodes.

\section{Robust MIMO $H_{\infty}$ control synthesis}

The aim of this section is to fulfill multiple-input multiple-output (MIMO) control objectives for the problem presented above. Considering the discrete perturbations induced by the doors and power sources along with the communication constraints, the robustness issue appears as critical. In addition, the use of a control approach that has been validated at the industrial level appears as an important issue in this application-oriented paper. The control setup is then first limited to a multi-objectives $H_{\infty}$ design as proposed in advanced control textbooks such as in Skogestad and Postlethwaite (2005) or Zhou and Doyle (1997).

\subsection{Steady-state and variation}

The first step is to rewrite the system dynamics (3.2) with a change of variables that allows for removing the known exogeneous inputs, in order to set the feedback on the tracking error. Defining the steady-state variables $\left\{x_{s s}, u_{s s}\right\}$ as constrained by the algebraic relationship:

$$
\left(A_{1}+A_{2} \operatorname{diag}\left(u_{s s}\right)\right) x_{s s}+B_{u} u_{s s}+B_{w} w=0
$$

the input that provides for a given reference state $x_{s s}$ is given by:

$$
u_{s s}=-\left(A_{2} \operatorname{diag}\left(x_{s s}\right)+B_{u}\right)^{-1}\left(A_{1} x_{s s}+B_{w} w\right)
$$

Introducing the variations $\left\{\tilde{x}(t) \doteq x(t)-x_{s s} ; \tilde{u}(t) \doteq u(t)-u_{s s}\right\}$, the regulated dynamics are obtained from (3.2) as:

$$
\begin{aligned}
\frac{d \tilde{x}}{d t} & =\left(A_{1}+A_{2} \operatorname{diag}\left(u_{S S}+\tilde{u}\right)\right)\left(x_{S s}+\tilde{x}\right)+B_{u}\left(u_{s S}+\tilde{u}\right)+B_{w} w \\
& \approx\left(A_{1}+A_{2} \operatorname{diag}\left(u_{s s}\right)\right) \tilde{x}+\left(A_{2} \operatorname{diag}\left(x_{s s}\right)+B_{u}\right) \tilde{u}
\end{aligned}
$$

and the aim of the control design is then to ensure that $\tilde{x}$ converges exponentially to 0 while satisfying some given closed-loop specifications.

\subsection{Mixed-sensitivity $H_{\infty}$ synthesis}

A mixed-sensitivity approach is chosen to tackle a multi-objective control design that can achieve performance, robustness and input boundary specifications. The main idea (see Skogestad and Postlethwaite (2005) for more details) is to introduce the closed-loop specifications as weights on the sensitivity 
functions prior to the $H_{\infty}$ norm minimization. Denoting the system sensitivity function as $S(s)$ and the complementary sensitivity as $T(s)$, the dynamic MIMO controller $K(s)$ is then designed to minimize the stack:

$$
\left\|\left[\begin{array}{c}
W_{p} S W_{d} \\
W_{u} K S \\
W_{t} T
\end{array}\right]\right\|_{\infty}
$$

where the specific choice of the weight is achieved as follows (considering $n$ rooms):

- the performance weight $W_{p}(s)$ is set as:

$$
W_{p}=\operatorname{diag}\left\{\frac{s / M+\omega_{B i}^{*}}{s+\omega_{B i}^{*} A}\right\} \text { with } i=1 \ldots n
$$

where $A=10^{-4}$ ensures an approximate integral action with $S(0) \approx 0, M=2$ and $\omega_{B i}^{*}$ is different for each output (a large value yields a faster response for the corresponding output);

- the input weight $W_{u}(s)$ is:

$$
W_{u}=\operatorname{diag}\left\{\frac{s}{s+\omega_{u}}\right\} \text { with } i=1 \ldots n
$$

to achieve tight control at low frequency, with $\omega_{u}$ being approximately the closed-loop bandwidth;

- the disturbance weight:

$$
W_{t}=\operatorname{diag}\left\{\frac{s+\omega_{B j}^{*} / M}{A s+\omega_{B j}^{*}}\right\} \text { with } j=1 \ldots n
$$

is introduced to reduce the impact of discrete events and measurements noise on the closed-loop.

The desired closed-loop response is then obtained thanks to an appropriate tuning of the performance weight with $\omega_{B i}^{*}$, of the input weight with $\omega_{u}$ and of the disturbance weight with $\omega_{B j}^{*}$.

EXAMPLE 5.1 For the 4-rooms test case, the control design parameters are set as:

$$
\omega_{B i}^{*}=\alpha_{p} \frac{\operatorname{mean}_{i}\left\{\rho_{a i r} V_{i} C_{v}\right\}}{\rho_{a i r} V_{i} C_{v}}, \quad \omega_{u}=\alpha_{u} \operatorname{mean}_{i}\left\{\omega_{B i}^{*}\right\}, \quad \omega_{B j}^{*}=\alpha_{t} \omega_{B i}^{*}
$$

to take into account the fact that faster control can be achieved in rooms with faster time constants (smaller volume) and to reduce the design problem to the choice of the three scalar parameters $\alpha_{p}, \alpha_{u}$ and $\alpha_{t}$. Setting the desired room temperature as $T d=273.15+\left[\begin{array}{llll}18 & 21 & 19 & 23\end{array}\right]^{T}(\mathrm{~K})$, and the controller parameters as $\alpha_{p}=1000, \alpha_{u}=10$ and $\alpha_{t}=1$, we obtain the closed-loop response presented in Figure 6. The high value of the parameters implies that all the desired bandwidth is available for control purposes, as the aim of this first test case is to get the best achievable performances without communication constraints. The higher sensitivity to doors actuation observed in rooms 1 and 3 is due to the fact that these rooms have a lower temperature then the adjacent ones, combined with the limitation to singledirection flows induced by the proposed 0D model (see Section 2).

The communication constraint are introduced thanks to the network model presented in Section 4. Considering wireless sensing capabilities and wired diffusers (connected in the underfloor space), the 


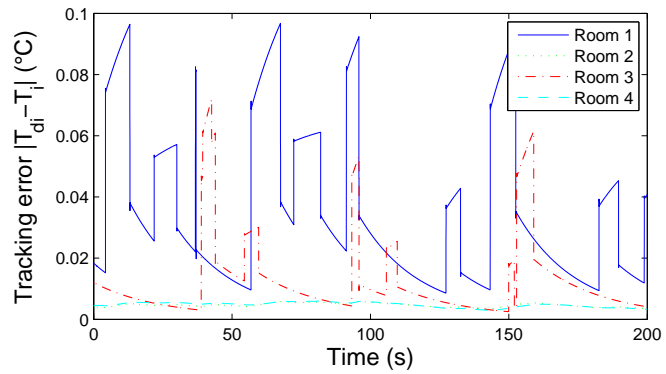

FIG. 6. Temperature tracking error without communication constraints

previous constraints (sampling, time-delay and packet losses) are introduced between the measurements and the controller. In order to ensure the closed-loop stability and give satisfying performances, the control parameters are set as $\alpha_{p}=0.1, \alpha_{u}=1$ and $\alpha_{t}=10$. The resulting temperature tracking errors are presented in Figure 7, for both synchronous and asynchronous modes. Note that the maximum errors in both cases are very similar $\left(\approx 2.5^{\circ} \mathrm{C}\right)$, which illustrates the robustness and performance limitation of the closed-loop to the network setup, and the weak influence of the mode choice in the WSN operation (synchronous or not).

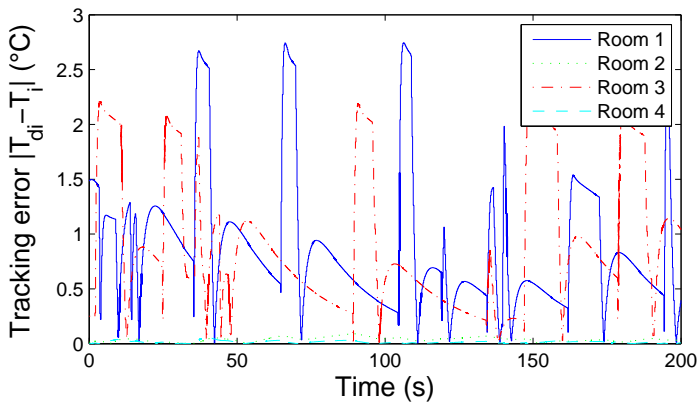

(a) Synchronous mode

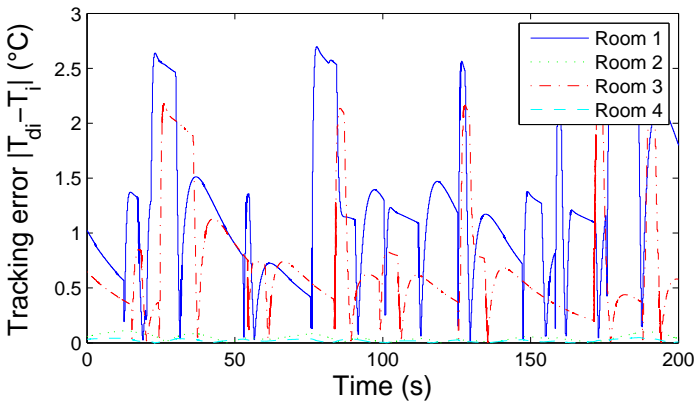

(b) Asynchronous mode

FIG. 7. Temperature tracking error with communication constraints (sensing over IEEE 802.15.4).

\section{Control with communication constraints}

According to the latter section, the simplified model writes as:

$$
\dot{x}=A x(t)+B u(t)+E w(t)
$$

where $x, u$ and $w$ are the state, the control input and the exogenous inputs, respectively. In order to embed specifications in the frequency domain, the filters $W_{u}(s)$ and $W_{p}(s)$ are added to the system, 
yielding the extended system:

$$
\begin{aligned}
& {\left[\begin{array}{c}
\dot{x} \\
\dot{x}_{e} \\
\dot{x}_{u}
\end{array}\right]=\underbrace{\left[\begin{array}{ccc}
A & 0 & 0 \\
-B_{p} & A_{p} & 0 \\
0 & 0 & A_{u}
\end{array}\right]}_{A_{g}}\left[\begin{array}{c}
x \\
x_{e} \\
x_{u}
\end{array}\right]+\underbrace{\left[\begin{array}{c}
B \\
B_{u}
\end{array}\right]}_{B_{g}} u+\underbrace{\left[\begin{array}{cc}
E \delta & 0 \\
0 & B_{p} \\
0 & 0
\end{array}\right]}_{E_{g}}\left[\begin{array}{c}
w \\
x_{r e f}
\end{array}\right]} \\
& {\left[\begin{array}{l}
e_{f}(t) \\
u_{f}(t)
\end{array}\right]=\underbrace{\left[\begin{array}{ccc}
-D_{p} & C_{p} & 0 \\
0 & 0 & C_{u}
\end{array}\right]}_{C_{g}}\left[\begin{array}{c}
x \\
x_{e} \\
x_{u}
\end{array}\right]+\underbrace{\left[\begin{array}{c}
0 \\
D_{u}
\end{array}\right]}_{D_{g}} u+\underbrace{\left[\begin{array}{cc}
0 & D_{p} \\
0 & 0
\end{array}\right]}_{F_{g}}\left[\begin{array}{c}
w \\
x_{r e f}
\end{array}\right]}
\end{aligned}
$$

where $\delta>1$ is a weight on the disturbances and:

$$
\left[\begin{array}{l|l}
A_{p} & B_{p} \\
\hline C_{p} & D_{p}
\end{array}\right]=W_{p}(s) \quad\left[\begin{array}{l|l}
A_{u} & B_{u} \\
\hline C_{u} & D_{u}
\end{array}\right]=W_{u}(s)
$$

are weights on the tracking error $e=x_{\text {ref }}-x$ and controlled input $u$. The aim of this section is to compute a controller which:

1. asymptotically stabilizes the system for any transmission delay value between $[0,0.2]$;

2. minimizes the $H_{\infty}$ norm of the transfer $\left[\begin{array}{c}w \\ x_{r e f}\end{array}\right] \rightarrow\left[\begin{array}{c}e_{f} \\ u_{f}\end{array}\right]$;

where $e_{f}$ and $u_{g}$ are filtered version of the tracking error $e$ and the control input $u$, defined by $E_{f}(s)=$ $W_{p}(s) E(s)$ and $U_{f}(s)=W_{u}(s) U(s)$ in the Laplace domain.

The considered controller is a particular state-feedback controller of the form:

$$
u(t)=K_{1} x(t)+K_{2} x_{e}(t)+K_{3} x_{u}(t)
$$

where the gains $K_{i}$ have to be determined. Note that since the control input depends on the states of the filters $W_{p}$ and $W_{u}$, the implemented controller includes the dynamical part of the weighting filters. To show this, let us rewrite equation (6.3) in the frequency domain:

$$
U(s)=K_{1} X(s)+K_{2}\left(s I-A_{p}\right)^{-1} B_{p} E(s)+K_{3}\left(s I-A_{u}\right)^{-1} B_{u} U(s)
$$

and equivalently:

$$
\begin{aligned}
U(s) & =H_{x}(s) X(s)+H_{r e f}(s) X_{r e f}(s), \text { with } \\
H_{x}(s) & \doteq\left(I-K_{3}\left(s I-A_{u}\right)^{-1} B_{u}\right)^{-1}\left(K_{1}-K_{2}\left(s I-A_{p}\right)^{-1} B_{p}\right) \\
H_{r e f}(s) & \doteq\left(I-K_{3}\left(s I-A_{u}\right)^{-1} B_{u}\right)^{-1} K_{2}\left(s I-A_{p}\right)^{-1} B_{p}
\end{aligned}
$$

The following result (bounded real lemma (Skelton et al., 1997)) is then used for controller gain synthesis.

LEMMA 6.1 There exists a control law that writes as (6.3) which asymptotically stabilizes the system (6.2) with an $H_{\infty}$ bound on the transfer $\left(w, x_{r e f}\right) \rightarrow\left(e_{f}, u_{f}\right)$ lower than $\gamma>0$ if and only if there exist matrices $P=P^{T} \succ 0$ and $Y$ of appropriate dimensions such that the linear matrix inequality (LMI):

$$
\left[\begin{array}{ccc}
A_{g} P+P A_{g}^{T}+B_{g} Y+Y^{T} B_{g}^{T} & E_{g} & P C_{g}^{T}+Y^{T} D_{g}^{T} \\
\star & -\gamma I & F^{T} \\
\star & \star & -\gamma I
\end{array}\right] \prec 0
$$


holds. Moreover, suitable controller gains are given by:

$$
\left[\begin{array}{lll}
K_{1} & K_{2} & K_{3}
\end{array}\right]=Y P^{-1}
$$

In order to characterize the stability of the closed-loop system with delays, we consider that the controller maintains the last measurement on its inputs until a new measure is available. We also consider that the same delay acts simultaneously on all the measurement. This is equivalent to a system output sampled at a time-varying sampling period. Hence, it is possible to consider results on stability analysis of systems with time-varying sampling period to analyze the system stability under communication constraints. It is important to mention that most of the works on such systems assume that the delay acts on the control input while in the current problem, the delay acts on the output of the model. However, since a state-feedback controller is considered, the problem is symmetric and available results are directly applicable.

An alternative approach is to consider the time-delay constraint directly during the controller synthesis, as proposed in (Seuret, 2009). The controller is then obtained thanks to the following Lemma.

LEMMA 6.2 The closed-loop system given by the interconnection of (6.2) and (6.3) is asymptotically stable for all delay belonging to $[0, \tau]$ if there exist matrices $P=P^{T} \succ 0, S=S^{T} \succ 0, R=R^{T} \succ 0$ and $N$ of appropriate dimensions such that the LMIs:

$$
\Pi_{1}+\tau \Pi_{2} \prec 0, \quad\left[\begin{array}{cc}
\Pi_{1} & \tau N \\
\star & -\tau R
\end{array}\right] \prec 0
$$

hold with:

$$
\begin{aligned}
& \Pi_{1}=M_{1}^{T} P M_{3}+M_{3}^{T} P M_{1}-M_{2}^{T} S M_{2}-N M_{2}-M_{2}^{T} N^{T} \\
& \Pi_{2}=M_{2}^{T} S M_{3}+M_{3}^{T} S M_{2}+M_{3}^{T} S M_{3}
\end{aligned}
$$

and $M_{1}=\left[\begin{array}{ll}I & 0\end{array}\right], M_{2}=\left[\begin{array}{ll}I & -I\end{array}\right]$ and $M_{3}=\left[\begin{array}{ll}A_{g} & B_{g} K\end{array}\right]$

EXAMPLE 6.1 The controllers obtained from the previous Lemmas are tested on the test case as follows.

Choosing $W_{u}(s)=s /\left(s+\omega_{1}\right), W_{p}(s)=\left(s / M s+\omega_{b}\right) /\left(s+A \omega_{b}\right), \delta=10, M_{s}=2, \omega_{b}=0.5, A=10^{-4}$, $\omega_{1}=10$ and using Lemma 6.1, we find $\gamma=0.93294$. However, such a small performance criterion is related to large values for eigenvalues and thus is responsible for a high sensitivity to delays. Thus in order to reduce this sensitivity, we impose $\gamma=10$ and get the following gains:

$$
\begin{gathered}
K_{1}=\left[\begin{array}{llll}
2.1921 & 0.0093 & 0.0009 & 0.0029 \\
0.0065 & 2.5798 & 0.0060 & 0.0003 \\
0.0010 & 0.0092 & 2.1173 & 0.0029 \\
0.0072 & 0.0013 & 0.0067 & 1.3593
\end{array}\right], \quad K_{2}=\left[\begin{array}{ccccc}
-1.1461 & -0.0009 & -0.0004 & 0.0002 \\
-0.0011 & -1.1673 & -0.0008 & -0.0002 \\
-0.0004 & -0.0011 & -1.1516 & 0.0001 \\
-0.0016 & -0.0005 & -0.0014 & -1.1115
\end{array}\right] \\
K_{3}=\left[\begin{array}{ccccc}
7.3908 & -0.0042 & -0.0011 & -0.0056 \\
-0.0054 & 7.6000 & -0.0054 & -0.0011 \\
-0.0010 & -0.0040 & 7.2683 & -0.0054 \\
-0.0029 & -0.0008 & -0.0029 & 5.4823
\end{array}\right]
\end{gathered}
$$

A bisection approach on the value of $\tau$ allows to find the maximal admissible value as $\tau_{\max }=0.2426 s$, which is sufficient for our application as the mean peak value for the delay is $0.2 s$. Using the latter controller, we obtain Figure 8(a). 


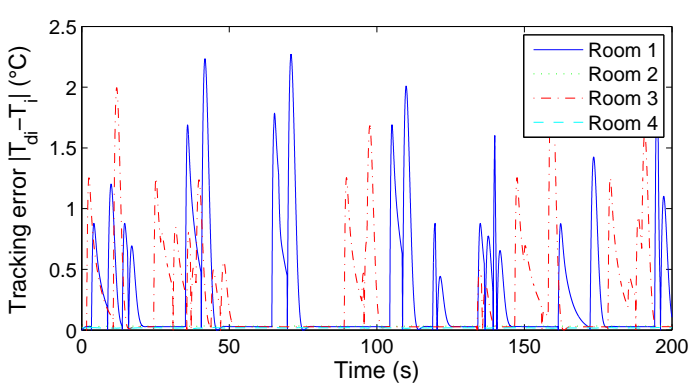

(a) Lemma 6.1

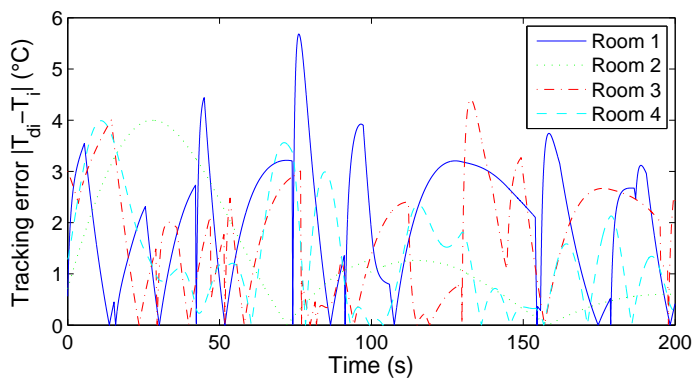

(b) Lemma 6.2

FIG. 8. Temperature tracking error with communication constraints.

On the other hand, when Lemma 6.2 is used for synthesis with the simplifications $S=\varepsilon_{S} P, \varepsilon_{s}=67$, $R=\varepsilon_{r} P, \varepsilon_{r}=34$ and $\tau=0.2 \mathrm{~s}$, we get errors evolving as depicted in Figure 8(b). We can clearly see that the maximal error values and the time needed to compensate a perturbation are significantly larger when the delay is included in the controller synthesis. This is related to the inherent conservatism associated with such methods.

\section{Conclusions}

In this work, we considered the problem of temperature regulation in intelligent buildings as the realtime control of an actuated UFAD process based on WSN measurements. A flexible model of the airflows was proposed based on the thermodynamics properties of the room control volume. Discrete events such as doors openings, people presence and the use of computers or printers were introduced as Markovian processes, which resulted in a hybrid nonlinear state-space description of the complete interconnected system. The deployment of a WSN with a star topology (IEEE 802.15.4) was detailed and shown to introduce communication constraints such as bit-rate limitations and time-delays. Synchronous and asynchronous communications are both considered.

Different MIMO $H_{\infty}$ controllers are then synthesized based on the linearized model dynamics and compared, in terms of temperature regulation performance. First, the controller design is achieved thanks to a mixed-sensitivity approach with performance, input weight and sensitivity to disturbance tuning parameters. It appears that the WSN has a strong impact on the closed-loop stability and necessitates to significantly reduce the performance expectations (the performance weight is decreased and the sensitivity to disturbance weight is increased). It is also noted that the choice between synchronous and asynchronous transmissions do not have a strong impact on the closed-loop system.

A second controller obtained thanks to the bounded-real lemma is then proposed, where the $H_{\infty}$ norm can be set explicitly. An appropriate choice of this norm allows to tune the sensitivity to timedelays and the maximum admissible delay is computed a posteriori. This controller appears to be the most efficient, in terms of temperature regulation (maximum peaks and response time).

Finally, the third controller is designed with a direct consideration of the maximum allowable delay in the gain synthesis. The resulting closed-loop performances appear to be worst than the other two design strategies, with a larger error and longer response time. This is probably related to the conservatism induced by such methods. 


\section{Funding}

The authors would like to thank Région Rhône-Alpes, GIPSA-lab (Grenoble Image Parole Signal Automatique laboratory) and Carnot-LSI (Logiciel et Systèmes Intelligents) institute for partial funding of this research. This work was also supported by the Swedish Research Council, the Swedish Strategic Research Foundation, and the Swedish Governmental Agency for Innovation System.

\section{References}

Bauman, F. and Daly, A. (2003). Underfloor Air Distribution Design Guide. ASHRAE, Atlanta, USA.

Fridman, E., Seuret, A., and Richard, J.P. (2004). Robust sampled-data stabilization of linear systems: An input delay approach. Automatica, 40, 1441-1446.

Giridhar, A. and Kumar, P.R. (2006). Toward a theory of in-network computation in wireless sensor networks. IEEE Communication Magazine, 44(4), 97-107.

Gladstone, C. and Woods, A. (2001). On buoyancy-driven natural ventilation of a room with a heated floor. Journal of Fluid Mechanics, 441(293-314).

IEEE (2006). IEEE Std 802.15.4-2996, September, Part 15.4: Wireless Medium Access Control (MAC) and Physical Layer (PHY) Specifications for Low-Rate Wireless Personal Area Networks (WPANs). URL http://www . ieee802.org/15.

Morton, B. (1959). Forced plumes. Journal of Fluid Mechanics, 5(1), 151-163.

Moteiv (2006). Tmote Sky Data Sheet. San Francisco, CA, sentilla edition. URL http://sentilla.com/files/pdf/eol/tmote-sky-datasheet.pdf.

Naghshtabrizi, P., Hespanha, J., and Teel, A. (2008). Exponential stability of impulsive systems with application to uncertain sampled-data systems. Systems \& Control Letters, 57, 378-385.

Park, P., Marco, P.D., Soldati, P., Fischione, C., and Johansson, K.H. (2009). A generalized markov chain model for effective analysis of slotted IEEE 802.15.4. In Proc. of the IEEE Conf. on Mobile Ad Hoc and Sensor Systems, 130-139. Macau SAR, P.R.C.

Sename, O. and Fattouh, A. (2007). Robust $h_{\infty}$ control of bilateral teleoperation systems under communication time-delay. In J. Loiseau and J. Chiasson (eds.), Applications of Time Delay Systems, LNCIS, 99-116. Springer, Berlin Heidelberg.

Seuret, A. (2009). Stability analysis for sampled-data systems with a time-varying period. In Proc. of the IEEE Conf. on Decision and Control, 8130-8135. Shanghai, China.

Skelton, R., Iwasaki, T., and Grigoriadis, K. (1997). A Unified Algebraic Approach to Linear Control Design. Taylor \& Francis, Abingdon.

Skogestad, S. and Postlethwaite, I. (2005). Multivariable Feedback Control: analysis and design. John Wiley $\&$ Sons, $2^{\text {nd }}$ edition.

Sonntag, R., Borgnakke, C., and Van Wylen, G. (1998). Fundamentals of Thermodynamics. John Wiley $\&$ Sons, $5^{\text {th }}$ edition. 
US department of energy (2008). EnergyPlus ${ }^{T M}$ Engineering Reference, University of Illinois and Ernest Orlando Lawrence Berkeley National Laboratory edition.

Willig, A., Matheus, K., and Wolisz, A. (2005). Wireless technology in industrial networks. Proceedings of the IEEE, 93(6), 1130-1151.

Witrant, E., D’Innocenzo, A., Sandou, G., Santucci, F., Di Benedetto, M., Isaksson, A., Johansson, K., Niculescu, S.I., Olaru, S., Serra, E., Tennina, S., and Tiberi, U. (2010a). Wireless ventilation control for large-scale systems: the mining industrial case. International Journal of Robust and Nonlinear Control, 20(2), 226-251.

Witrant, E., Mocanu, S., and Sename, O. (2009). A hybrid model and MIMO control for intelligent buildings temperature regulation over WSN. In Proc. of the IFAC Workshop on Time Delay Systems. Sinaia, Romania.

Witrant, E., Park, P., and Johansson, M. (2010b). Time-delay estimation and finite-spectrum assignment for control over multi-hop wsn. In S. Mazumder (ed.), Wireless Network Based Control. Springer, Berlin Heidelberg.

Yih, C.S. (1969). Stratified flows. Annual Review of Fluid Mechanics, 1, 73-110.

Zhou, K. and Doyle, J. (1997). Essentials Of Robust Control. Prentice Hall, Upper Saddle River. 